\title{
Eigenvalue spectra of asymmetric random matrices for multicomponent neural networks
}

\author{
Yi Wei \\ Cold Spring Harbor Laboratory, Cold Spring Harbor, New York 11724, USA
}

(Received 31 January 2012; published 15 June 2012)

\begin{abstract}
This paper focuses on large neural networks whose synaptic connectivity matrices are randomly chosen from certain random matrix ensembles. The dynamics of these networks can be characterized by the eigenvalue spectra of their connectivity matrices. In reality, neurons in a network do not necessarily behave in a similar way, but may belong to several different categories. The first study of the spectra of two-component neural networks was carried out by Rajan and Abbott [Phys. Rev. Lett. 97, 188104 (2006)]. In their model, neurons are either "excitatory" or "inhibitory," and strengths of synapses from different types of neurons have Gaussian distributions with different means and variances. A surprising finding by Rajan and Abbott is that the eigenvalue spectra of these types of random synaptic matrices do not depend on the mean values of their elements. In this paper we prove that this is true even for a much more general type of random neural network, where there is a finite number of types of neurons and their synaptic strengths have correlated distributions. Furthermore, using the diagrammatic techniques, we calculate the explicit formula for the spectra of synaptic matrices of multicomponent neural networks.
\end{abstract}

DOI: 10.1103/PhysRevE.85.066116

PACS number(s): 84.35.+i, 02.50.-r, 05.10.-a

\section{INTRODUCTION}

In neuroscience, interconnections of neurons are often represented by synaptic matrices whose elements are drawn from a certain random matrix ensemble [1,2]. Knowing the distribution of eigenvalues of these random matrices is very important in studying spontaneous activities and evoked responses of the network. To calculate the eigenvalue distribution of these matrices, it is often necessary to work with asymmetric (non-Hermitian) random matrix theory, which has been successfully applied to many fields of physics and interdisciplinary sciences, e.g., the phase diagram of QCD $[3,4]$, nuclear decay and resonances in multichannel chaotic scattering [5], and neural networks [1,2,6].

A prominent result of asymmetric random matrix theory is Girko's circle law [7]. In its variation with partial symmetry, the circle becomes an ellipse [6]. These classic results, however, cannot be directly applied to realistic neural network models where neurons do not behave in the same way $[1,8,9]$. Assume there are $N$ number of neurons and let $W$ be the synaptic matrix. In the model of Rajan and Abbott [1], there are $f N$ numbers of neurons which are "excitatory," and all others are "inhibitory." To model this neural network, elements in $f N$ columns of $W$ are sampled from a Gaussian distribution with mean $\mu_{E} / \sqrt{N}$ and variance $\sigma_{E}^{2} / N$ and elements in the remaining $(1-f) N$ columns of $W$ are Gaussian variables with mean $\mu_{I} / \sqrt{N}$ and variance $\sigma_{I}^{2} / N$. Therefore, the synaptic matrix has the structure $W=J \Lambda+$ $M$, where $J$ is drawn from the real Ginibre ensemble [10] such that $\left\langle J_{i j}\right\rangle=0,\left\langle J_{i j}^{2}\right\rangle=1 / N$, and $\Lambda=\operatorname{diag}\left(\sigma_{E} I_{f N}, \sigma_{I} I_{(1-f) N}\right)$, where $I_{f N}$ and $I_{(1-f) N}$ are identity matrices of dimension $f N$ and $(1-f) N$, respectively. $M$ is a constant matrix whose elements are the mean strengths of the synapses. Since there are two types of synapses, every row of $M$ is identical, and in each row, the first $f N$ elements are equal to $\mu_{E} / \sqrt{N}$ and the remaining $(1-f) N$ elements are equal to $\mu_{I} / \sqrt{N}$. In particular, $M$ is chosen to be in a "balanced" situation such that $f \mu_{E}+(1-f) \mu_{I}=0[11,12]$. To confine eigenvalues inside a unit circle, a second constraint [1] is introduced which requires that the strengths of the synapses attached to each neuron independently sum to zero. It is found in Ref. [1] that, in the limit $N \rightarrow \infty$, modifying the mean strengths of excitatory and inhibitory synapses has no effect on the eigenvalue spectra of the synaptic matrices. Therefore, the spectrum of $W$ is identical to that of $J \Lambda$.

It is natural to wonder why the "mean" strength matrix $M$ has no effect on the spectra. Moreover, in real biological neural systems, several different types of neurons may connect with each other to form a multicomponent network [8,9]. Distributions of synaptic strengths of different types of neurons are distinct [13] and could be non-Gaussian [13,14]. The dynamics of this network therefore depends on properties of each type of neuron. It is interesting to find whether or not the eigenvalue density of this type of networks depends on the mean value of each individual type of synapse. These questions are addressed in Sec. II. One of the main results of this paper shows that, even without the second constraint in Ref. [1] and when synaptic strengths have certain nonGaussian distributions, the spectrum of the network still does not depend on the mean synaptic strengths.

Aside from biological motivations, the eigenvalue problem of random plus fixed matrices has been a research topic in both random matrix theory and condensed matter physics for a long time [15-18]. A different point of view of the problem in this paper is the following: how is the density function of large random matrix $J \Lambda$ perturbed by the rank- 1 constant matrix $M$ ? Note that random matrix $J \Lambda$ is not of Wigner type, nor are its elements independently and identically distributed (iid). Therefore this paper provides results to similar problems studied in Refs. [17,18].

Furthermore, finding the eigenvalue density of random matrices of the form $J \Lambda$, where $J$ is drawn from a random matrix ensemble and $\Lambda$ is a fixed matrix, has been an interesting topic in random matrix theory and mesoscopic physics [19]. When $J$ is drawn from the circular unitary ensemble (CUE), an exact result is given in Ref. [20], and the large- $N$ limit is calculated in Ref. [21]. In Sec. III, we calculate the density function of $J \Lambda$ where $J$ belongs to the real Ginibre ensemble using the 
method introduced in Refs. [22,23]. Discussion and remarks are made in the last section.

\section{SYNAPTIC STRENGTH OF NON-GAUSSIAN DISTRIBUTIONS}

Let the $N \times N$-dimensional real matrix $W$ be the synaptic matrix of an $N$-neuron network. Assume there are $m$ types of neurons and the $i$ th type of neuron has a population of $f_{i} N$, $\sum_{i=1}^{m} f_{i}=1$. Define a constant diagonal matrix

$$
\Lambda=\operatorname{diag}\left(\sigma_{1} I_{f_{1} N}, \ldots, \sigma_{m} I_{f_{m} N}\right)>0,
$$

where $I_{f_{i} N}$ is the $f_{i} N$-dimensional identity matrix. Let $v$ be an $N$-dimensional row vector with the following form,

$$
v=(\underbrace{\mu_{1}, \ldots, \mu_{1}}_{f_{1} N}, \underbrace{\mu_{2}, \ldots, \mu_{2}}_{f_{2} N}, \ldots, \underbrace{\mu_{m}, \ldots, \mu_{m}}_{f_{m} N}),
$$

where $\mu_{i}$ is the mean strength of the synapses from neurons of the $i$ th type. Define the $N \times N$-dimensional mean matrix $M$, whose rows are all equal to $v$. The synaptic matrix $W$ in our model takes the form

$$
W=J \Lambda+M,
$$

where $J$ is an $N \times N$-dimensional real random matrix drawn from the ensemble

$$
P(J)=\frac{1}{Z} \exp \left[-N \operatorname{tr} V\left(J J^{T}\right)\right],
$$

where $V$ is an arbitrary function and $Z$ is the normalization constant. The case $V(x)=x / 2$ corresponds to the Ginibre ensemble where elements of $J$ are statistically independent Gaussian variables. By symmetry, the mean of $J_{i j}$ vanishes, $\left\langle J_{i j}\right\rangle=0$. The variance of $J_{i j}$ is determined by $V$, which is normalized to be $1 / N$, i.e., $\left\langle J_{i j}^{2}\right\rangle=1 / N$.

Synaptic matrix $W$ defined in Eq. (2.3) has $m$ column blocks. Each block corresponds to one type of neuron. By construction, elements of $W$ have the following statistical properties,

$$
\operatorname{Var}\left(W_{i j}\right)=\Lambda_{j j}^{2} / N \quad \text { and } \quad\left\langle W_{i j}\right\rangle=v_{j},
$$

i.e., the $i$ th type of synaptic strength has variance $\sigma_{i}^{2} / N$ and mean $\mu_{i}$. The matrix $M$ has a column block structure similar to that defined in Ref. [1]. Following Ref. [1], we also chose to put the synapses in the balanced situation, i.e.,

$$
\sum_{i=1}^{m} f_{i} \mu_{i}=0
$$

We want to know the eigenvalue density $\rho(x, y)$ of $W$ in the limit $N \rightarrow \infty$, with fixed $f$ 's. Let $z=x+i y$, the density $\rho(x, y)$ is related to the Green's function $G_{W}(z, \bar{z})$ as

$$
\begin{aligned}
\rho(x, y) & =\frac{1}{\pi} \frac{\partial}{\partial \bar{z}} G_{W}(z, \bar{z}), \quad \text { where } \\
G_{W}(z, \bar{z}) & =\frac{1}{N}\left\langle\operatorname{tr}_{N} \frac{1}{z-W}\right\rangle_{J} .
\end{aligned}
$$

In the above formula, $\langle\cdots\rangle_{J}$ means averaging over the ensemble Eq. (2.4) of matrix $J$. We write the Green's function as $G_{W}(z, \bar{z})$ to emphasize it is not analytic on a two-dimensional region of the $(x, y)$ plane, more details can be found in Ref. [23]. This region is called the support of the density function because we have $\frac{\partial}{\partial \bar{z}} G_{W}(z, \bar{z}) \neq 0$. Since we will be dealing with both $N \times N$ - and $2 N \times 2 N$-dimensional matrices, to remove ambiguity, we use $\operatorname{tr}_{N}$ as the trace operator for $N \times N$ matrices. We work on asymmetric random matrices with the methods introduced in Refs. [22,23]. For consistency, we adopt the notation convention of Ref. [22] in the remainder of this paper. We define a $2 N \times 2 N$-dimensional matrix:

$$
\mathcal{Z}=\left(\begin{array}{ll}
z & \lambda \\
\lambda & \bar{z}
\end{array}\right)
$$

For asymmetric matrix $W$, we define the resolvent (matrix valued Green's function) $\mathcal{G}_{W}[22,23]$ as

$$
\mathcal{G}_{W}(\mathcal{Z})=\left(\begin{array}{ll}
\mathcal{G}_{1} & \mathcal{G}_{2} \\
\mathcal{G}_{3} & \mathcal{G}_{4}
\end{array}\right)=\left\langle\left[\mathcal{Z}-\left(\begin{array}{ll}
W & \\
& W^{T}
\end{array}\right)\right]_{2 N \times 2 N}^{-1}\right\rangle_{J}
$$

Introducing the self-energy $\Sigma_{W}$, we have

$$
\mathcal{G}_{W}(\mathcal{Z})=\frac{1}{\mathcal{Z}-\Sigma_{W}} .
$$

The Green's function in Eq. (2.7) can be found from $\mathcal{G}_{W}[22,23]$,

$$
G_{W}(z, \bar{z})=\lim _{\lambda \rightarrow 0, N \rightarrow \infty} \frac{1}{N} \operatorname{tr}_{N} \mathcal{G}_{1},
$$

where the limit $N \rightarrow \infty$ is taken before $\lambda \rightarrow 0$. Similarly, as in Eqs. (2.7)-(2.11), we define $\mathcal{G}_{J \Lambda}, \Sigma_{J \Lambda}$, and $G_{J \Lambda}$. Introducing a constant matrix

$$
\mathcal{M}=\left(\begin{array}{ll}
M & \\
& M^{T}
\end{array}\right),
$$

we have the relation

$$
\mathcal{G}_{W}(\mathcal{Z})=\mathcal{G}_{J \Lambda}(\mathcal{Z}-\mathcal{M})=\frac{1}{\mathcal{Z}-\mathcal{M}-\Sigma_{J \Lambda}} .
$$

It is impossible to calculate $\Sigma_{J \Lambda}$ explicitly for arbitrary $V$. But for our purpose it is sufficient to know its basic structure. Without loss of generality, assume $V(x)=x / 2+\cdots$, so that we can expand $\operatorname{tr} V\left(J J^{T}\right)$ as

$$
\operatorname{tr}_{N} V\left(J J^{T}\right)=\frac{1}{2} \operatorname{tr}_{N} J J^{T}+g_{2} \operatorname{tr}_{N}\left(J J^{T}\right)^{2}+g_{3} \operatorname{tr}_{N}\left(J J^{T}\right)^{3}+\cdots .
$$

We expand the higher order terms in Eq. (2.14) and use the quadratic term to calculate the ensemble averages, denoted by \langle\rangle$_{0}$. Let $\bar{W}=J \Lambda$. Then because of the presence of matrix $\Lambda$, we have

$$
\left\langle\bar{W}_{a b} \bar{W}_{c d}^{T}\right\rangle_{0}=\frac{\Lambda_{b b}^{2}}{N} \delta_{a d} \delta_{b c}, \quad\left\langle\bar{W}_{a b}\right\rangle_{0}=0 .
$$

The self-energy $\Sigma_{J \Lambda}$ can be written in terms of the cumulants of $P(J)$, i.e., $\Gamma_{2 k}, k=1,2, \ldots$. And it is well known that in the limit $N \rightarrow \infty$, to leading order in $\frac{1}{N}$, each of these cumulants is the sum of all connected planar diagrams with $k$ external $J$ 's and $J^{T}$ 's. All diagrams which contribute to $\Sigma_{J \Lambda}$ are also planar diagrams, as shown in Fig. 1. By Eq. (2.15), the self-energy $\Sigma_{J \Lambda}$ has the following structure:

$$
\Sigma_{J \Lambda}=\left(\begin{array}{cc}
\Sigma_{1} & \Sigma_{2} \\
\Sigma_{3} & \Sigma_{4}
\end{array}\right)=\frac{1}{N}\left(\begin{array}{cc}
0 & a I_{N} \\
b \Lambda^{2} & 0
\end{array}\right),
$$




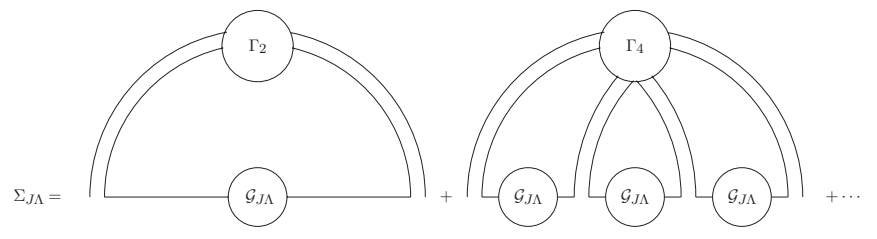

FIG. 1. Contributions of the quadratic cumulant $\Gamma_{2}$ and the quartic cumulant $\Gamma_{4}$ to the self-energy $\Sigma_{J \Lambda}$.

where scalars $a$ and $b$ are functions of $z$ and $\bar{z}$ and are determined by $V$ and $\Lambda$ is defined in Eq. (2.1). In the Appendix, we prove that $G_{W}=G_{J \Lambda}$. This fact, together with Eq. (2.7), completes the proof that the eigenvalue spectrum of $W$ is identical to that of the random matrix $J \Lambda$, as discovered in Ref. [1] when $J$ belongs to the Ginibre ensemble.

In Fig. 2, we compare the eigenvalue spectra of $W=$ $J \Lambda+M$ and $W=J \Lambda$. In both cases $J$ is drawn from a non-Gaussian ensemble. All spectra are generated by Monte Carlo simulations. Since $\rho(x, y)=\rho(|z|)$, where $z=x+i y$, it is sufficient to show the dependence of eigenvalue density function on radius $|z|$. We find these functions match quite well. In comparison, we replace $M$ with a constant matrix, which does not have the column structure, and find the density function is rather different.

Next, we test our result on the two-component Gaussian network of Ref. [1]. Denote the weight matrix by $W=J \Lambda+$ $M$ and follow the brief discussion in the Introduction. We fix
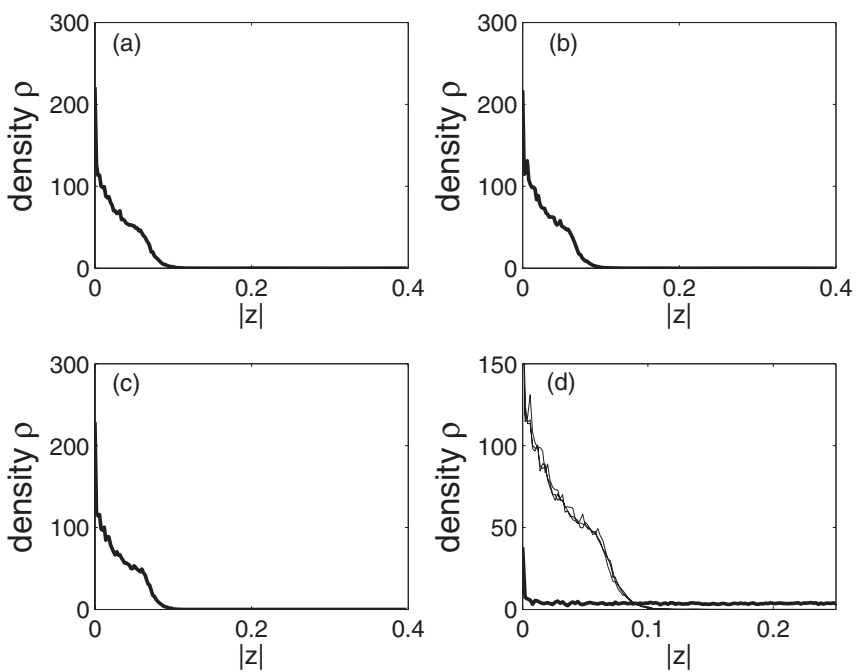

FIG. 2. Density $\rho$ of eigenvalues as a function of radius $|z|$ in the complex plane. Simulations are run for random matrices of dimension $N=200$, drawn from the ensemble defined in Eq. (2.4) with $V(x)=x+x^{2}$. There are four types of neurons in the network, with $f=(0.1,0.2,0.3,0.4)$ and $\Lambda=\operatorname{diag}\left(0.5 I_{20}, 1.0 I_{40}, 1.5 I_{60}, 2.0 I_{80}\right)$. (a) $\mu=(10,30,30,-40)$. (b) $\mu=(1,3,3,-4)$. (c) $\mu=(0,0,0,0)$, i.e., $M=0$. (d) Density functions in panels (a)-(c) are drawn in the same plane. We find the bulk of the three functions are very similar, which shows that even when elements of weight matrix $M$ are of order higher than $1 / \sqrt{N}$, the density function of $J \Lambda+M$ still converges to that of $J \Lambda$ as $N \rightarrow \infty$. This is due to the column structure of $M$. In comparison, we show in panel (d) the density function of $W=J \Lambda+M$, where $M$ is a constant matrix whose elements are randomly chosen from uniform distribution on $[0,1]$.

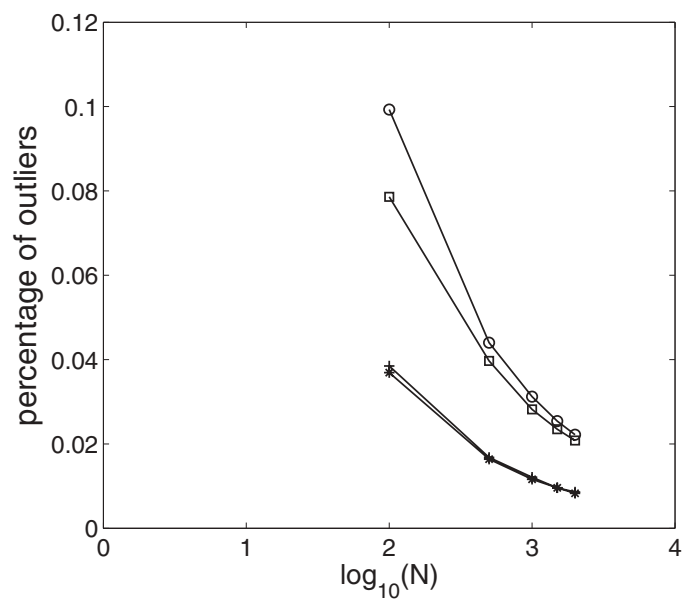

FIG. 3. Percentage of eigenvalue outliers of two-component Gaussian network $W$ decays as $N$ increases. For $f=0.2$, elements in $f N$ columns of $W$ have Gaussian distribution with variance $\frac{2}{N}$ and mean $\mu_{e}$. Other elements have Gaussian distribution with variance $\frac{1}{N}$ and mean $\mu_{i}$. From top to bottom, $\left(\mu_{e}, \mu_{i}\right)$ are $\left(1,-\frac{1}{4}\right),\left(\frac{1}{\sqrt{N}},-\frac{1}{4 \sqrt{N}}\right)$, $\left(\frac{1}{N},-\frac{1}{4 N}\right)$, and $(0,0)$, respectively.

$f=0.2$ and choose the variance of the Gaussian distributions for excitatory and inhibitory synaptic strengths to be $\frac{2}{N}$ and $\frac{1}{N}$, respectively. The only constraint on the mean strength matrix $M$ is the balance condition. As in the previous example and already pointed out in Ref. [1], the bulk of the spectra of $W$ with different $M$ matrices are almost identical. From numerical simulations, it appears that when $M$ has larger elements, there are more eigenvalues outside the support of the spectrum; see Eq. (3.6) for the definition. To show this is a finite-size effect, we calculate the percentage of outliers, for different matrix size $N$. It shows in Fig. 3 that, for all values of $M$, as $N \rightarrow \infty$, the percentages of outliers for $W=J \Lambda+M$ approach those of $W=J \Lambda$.

\section{SYNAPTIC STRENGTH OF GAUSSIAN DISTRIBUTION}

In this section, we calculate the eigenvalue density of a multicomponent Gaussian network. Assume there are $m$ types of neurons in the network and synaptic strengths have different Gaussian distributions. From the previous section we know the density functions of $J \Lambda+M$ and $J \Lambda$ are identical when $M$ has the column structure and satisfies the balance condition, even without the additional constraint of Ref. [1]. Therefore, the spectrum of this network is the density function $\rho(x, y)$ of the following random matrix,

$$
W=J \Lambda
$$

where $J$ is drawn from the real Ginibre ensemble, i.e., $V(x)=$ $\frac{1}{2} x$ in Eq. (2.4), and $\Lambda$ is defined in Eq. (2.1). The case $m=2$ is solved in Ref. [1] with the method in Ref. [6]. For $m>2$, we find the technique developed in Refs. [22,23] is more convenient. Define an operator $\overline{\operatorname{tr}}_{N}$ which, when it acts on an $N \times N$ matrix $A$, gives $\operatorname{tr}_{N} A=\operatorname{tr}_{N} A \Lambda^{2}$. By Eq. (2.15), the equation for the one particle irreducible (1PI) self-energy $\Sigma_{W}$ 


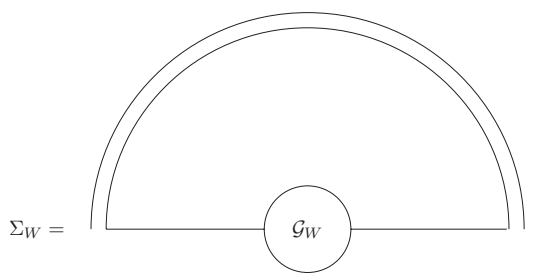

FIG. 4. Self-energy $\Sigma_{W}$ is related to resolvent $\mathcal{G}_{W}$ by Eq. (3.2).

(Fig. 4) is

$$
\Sigma_{W}=\left(\begin{array}{cc}
\Sigma_{1} & \Sigma_{2} \\
\Sigma_{3} & \Sigma_{4}
\end{array}\right)=\frac{1}{N}\left(\begin{array}{cc}
0 & \overline{\operatorname{tr}}_{N} \mathcal{G}_{2} \cdot I_{N} \\
\operatorname{tr}_{N} \mathcal{G}_{3} \cdot \Lambda^{2} & 0
\end{array}\right) .
$$

Note that the above matrix has the structure outlined in Eq. (2.16). The generalized Green's function $\mathcal{G}_{W}$ [defined in Eq. (2.9)] is related to $\Sigma_{W}$ by the Schwinger-Dyson equation, Eq. (2.10),

$$
\mathcal{G}_{W}=\left(\begin{array}{ll}
\mathcal{G}_{1} & \mathcal{G}_{2} \\
\mathcal{G}_{3} & \mathcal{G}_{4}
\end{array}\right)=\left(\begin{array}{ll}
z-\Sigma_{1} & \lambda-\Sigma_{2} \\
\lambda-\Sigma_{3} & \bar{z}-\Sigma_{4}
\end{array}\right)_{2 N \times 2 N}^{-1} .
$$

From Eqs. (3.2) and (3.3), we get the following equation for an unknown variable $p=\frac{1}{N} \overline{\operatorname{tr}}_{N} \mathcal{G}_{2} \cdot \frac{1}{N} \operatorname{tr}_{N} \mathcal{G}_{3}$,

$$
\sum_{i=1}^{m} \frac{f_{i} \sigma_{i}^{2}}{|z|^{2}-p \sigma_{i}^{2}}=1 \text {. }
$$

Equation (3.4) has multi-number of solutions. The correct one for our problem is the one satisfying the boundary condition

$$
\left.p\right|_{|z|^{2}=0}=-1 \text {. }
$$

The boundary of spectrum is determined by the transition point $p=0$ [22], which corresponds to the circle with radius $|z|_{B}$, such that

$$
|z|_{B}^{2}=\sum_{i=1}^{m} f_{i} \sigma_{i}^{2}
$$

The disk region defined by $|z| \leqslant|z|_{B}$ is the support of the spectrum. Off the support, we always have $p=0$. In the case $m=2$, the formula in Eq. (3.6) gives the same result for the spectrum boundary obtained in Ref. [1] by solving a saddle-point equation. From Eq. (2.11), the Greens's function $G_{W}(z, \bar{z})$ is given by the following formula:

$$
G_{W}(z, \bar{z})= \begin{cases}\bar{z} \sum_{i=1}^{m} \frac{f_{i}}{|z|^{2}-p \sigma_{i}^{2}}, & |z|^{2} \leqslant|z|_{B}^{2}, \\ \frac{1}{z}, & |z|^{2}>|z|_{B}^{2} .\end{cases}
$$

From Eq. (3.4), when on the support of spectrum, we get

$$
\frac{\partial p}{\partial|z|^{2}}=\frac{\sum_{i=1}^{m} \frac{f_{i} \sigma_{i}^{2}}{\left(|z|^{2}-p \sigma_{i}^{2}\right)^{2}}}{\sum_{i=1}^{m} \frac{f_{i} \sigma_{i}^{4}}{\left(|z|^{2}-p \sigma_{i}^{2}\right)^{2}}}
$$

Finally, by Eq. (2.7), we get the eigenvalue density of $W$ :

$$
\rho(x, y)= \begin{cases}\frac{1}{\pi}\left(|z|^{2} \frac{\partial p}{\partial|z|^{2}}-p\right) \sum_{i=1}^{m} \frac{f_{i} \sigma_{i}^{2}}{\left(|z|^{2}-p \sigma_{i}^{2}\right)^{2}}, & |z|^{2} \leqslant|z|_{B}^{2}, \\ 0, & |z|^{2}>|z|_{B}^{2} .\end{cases}
$$

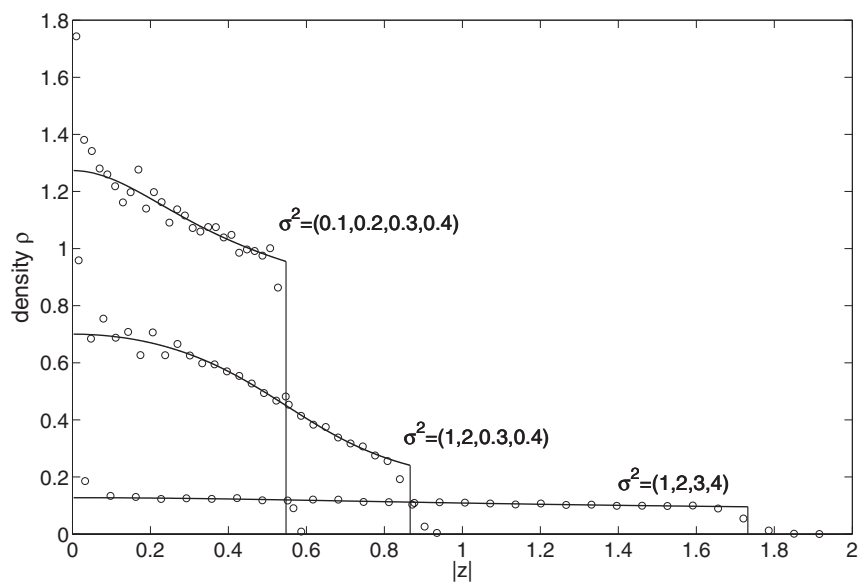

FIG. 5. Density $\rho$ of eigenvalues as a function of radius in the complex plane $|z|$, for $N=400$. The solid lines are the analytic results by Eq. (3.9) and the symbols are numerical simulations. The figure shows results for different sets of variances $\sigma^{2} / N$ with fixed population $f=(0.1,0.2,0.3,0.4)$.

We introduce the notation $\left\langle\sigma^{a}\right\rangle=\sum_{i}^{m} f_{i} \sigma_{i}^{a}$, where $a$ is a constant. From Eq. (3.9), we find the eigenvalue density at the center and boundary of the spectrum:

$$
\rho(0)=\frac{1}{\pi}\left\langle\sigma^{-2}\right\rangle \text { and } \rho\left(|z|_{B}\right)=\frac{1}{\pi} \frac{\left\langle\sigma^{2}\right\rangle}{\left\langle\sigma^{4}\right\rangle} \text {. }
$$

When $m=1$, from Eqs. (3.4) and (3.9) we easily recover the well-known result for the Ginibre ensemble [10]. For $m=2$, choosing the solution for the quadratic equation, Eq. (3.4), satisfying condition (3.5), and then from Eq. (3.9), we successfully recover the results obtained in Ref. [1]. For large $m$, it is hardly possible to have an analytic solution for Eq. (3.4). But it is very simple to find the numerical solution for this algebraic equation. It turns out that there is always only one solution on $[-1,0]$, which is just what we need according to the boundary conditions.

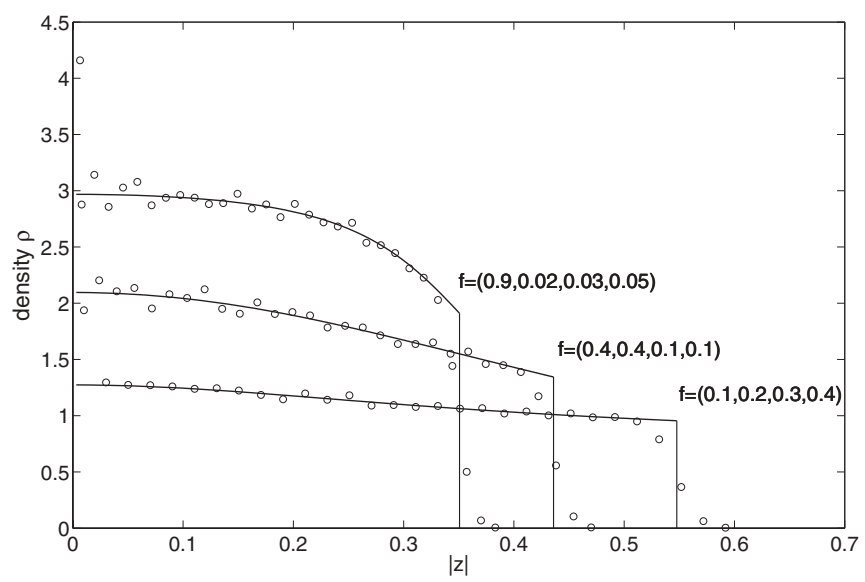

FIG. 6. Density $\rho$ of eigenvalues as a function of radius in the complex plane $|z|$, for $N=400$. The solid lines are the analytic results obtained by Eq. (3.9) and the symbols are numerical simulations. The figure shows results for different sets of population with fixed variances $\sigma^{2}=(0.1,0.2,0.3,0.4) / N$. 
In Fig. 5, we compare the density function in Eq. (3.9) with numerical simulations for synaptic strengths with different variances $\sigma$ 's but the same $f$ 's. In Fig. 6, we let the $f$ 's change but keep the $\sigma$ 's fixed. In both cases, we observe a very good match between numeric data and analytical results. The only significant deviation happens near $|z|=0$. In fact, this deviation already appears when $\Lambda=I_{N}$ and it is shown to be due to the finite-size effect [6].

\section{DISCUSSION}

In the first part of this paper, we show that modifying the mean strengths of synapses of a neural network does not change the density function of the synaptic matrix even when there are several types of neurons and the strengths of their synaptic connections have correlated distributions.

In Eq. (2.4), the ensemble of random matrix $J$ is chosen to be $O(N)$ invariant so that all elements of random matrix $J$ have the same distribution. Differences between different types of neurons are introduced only by $\Lambda$ and $M$. In fact, we can draw the synaptic matrix $W$ from more general ensembles. As long as Eq. (2.15) holds and $M$ has the column block structure, eigenvalue spectra of $W$ will not depend on $M$.

We therefore prove that the density functions of large random matrices described by Eqs. (2.3) and (2.4) are not changed by perturbations of the rank-1 matrix $M$. This type of random matrices are not of Wigner type or have iid elements as in Refs. [17,18].

It is its structure that makes $M$ irrelevant to the eigenvalue density function. In reality, we may need to choose the mean value of synaptic connections to be of the same order of their fluctuations, i.e., $\left\langle M_{i j}\right\rangle \propto N^{-1 / 2}$. But this is not necessary in our proof. If the balance condition is not imposed, the eigenvalue spectra will be identical to the balanced case except the eigenvalues at zero will be shifted $[1,17]$.

In the second part of this paper we calculate the density function of random matrices of the form $J \Lambda$, where $J$ belongs to the Ginibre ensemble. These matrices describe random networks with multiple independent components. We find closed formulas for the eigenvalue density at both the center and the boundary of the spectrum in terms of variances of synaptic strengths.

When $J$ is drawn from the ensemble in Eq. (2.4) and $\Lambda=I_{N}$, we know by the single-ring theorem [24-26] that the support of the eigenvalue spectrum is either a disk or an annulus. It will be interesting to find out whether or not the single-ring theorem still holds when $\Lambda$ is diagonal but not proportional to $I_{N}$. Equation (3.6) shows when $J$ has Gaussian distribution the support of the spectrum is always a disk of radius $|z|_{B}$, but never an annulus. This indeed agrees with the single-ring theorem. Clearly, to prove the single-ring theorem for $J \Lambda$, where $J$ belongs to the general ensemble defined in Eq. (2.4), we need to take different approaches. Work on this topic is currently in process.

\section{ACKNOWLEDGMENTS}

I am grateful to Professor R. T. Scalettar for helpful communication on numerical methods and giving me his Monte Carlo code. I also thank Dr. K. Rajan for drawing my interest to this research. This work was supported by the Swartz Foundation.

\section{APPENDIX}

In this section we show that in the large- $N$ limit, due to the structure of $\mathcal{M}$, the Green's function $G_{W}$ defined in Eq. (2.11) equals to $G_{J \Lambda}$ defined similarly for $J \Lambda$, i.e.,

$$
G_{W}=G_{J \Lambda}+O\left(N^{-1}\right) .
$$

Step 1. Define $D=\left(\bar{z}-M^{T}\right)(z-M)-a b \Lambda^{2}$, where $a$ and $b$ are scalars shown in Eq. (2.16). By Eq. (2.13), $\mathcal{G}_{W}$ can be written as

$$
\mathcal{G}_{W}=\left(\begin{array}{cc}
D^{-1}\left(\bar{z}-M^{T}\right) & a D^{-1} \\
* & (z-M) D^{-1}
\end{array}\right) .
$$

Here we used the fact that the (12)-element of $\Sigma_{J \Lambda}$ is proportional to the identity matrix and the following formula from linear algebra:

$$
\left(\begin{array}{cc}
E & F \\
G & H
\end{array}\right)^{-1}=\left(\begin{array}{cc}
E^{-1}+E^{-1} F X^{-1} G E^{-1} & -E^{-1} F X^{-1} \\
-X^{-1} G E^{-1} & X^{-1}
\end{array}\right)
$$

where $X=H-G E^{-1} F$.

Step 2. Let $\mathbb{I}_{p q}$ be a $p \times q$-dimensional matrix with all elements equal to 1 and let $M_{i}=f_{i} N$ for $i=1, \ldots, m$. Then $D^{-1}$ has the following $m \times m$ block structure:

$D^{-1}$

$$
=\left(\begin{array}{cccc}
a_{1}+b_{11} \mathbb{I}_{M_{1} M_{1}} & b_{12} \mathbb{I}_{M_{1} M_{2}} & \cdots & b_{1 m} \mathbb{I}_{M_{1} M_{m}} \\
b_{21} \mathbb{I}_{M_{2} M_{1}} & a_{2}+b_{22} \mathbb{I}_{M_{2} M_{2}} & \cdots & b_{2 m} \mathbb{I}_{M_{2} M_{m}} \\
\vdots & \vdots & \ddots & \vdots \\
b_{m 1} \mathbb{I}_{M_{m} M_{1}} & b_{m 2} \mathbb{I}_{M_{m} M_{2}} & \cdots & a_{m}+b_{m m} \mathbb{I}_{M_{m} M_{m}}
\end{array}\right) \text {, }
$$

where $a_{i}=1 /\left(|z|^{2}-a b \lambda_{i}^{2}\right), b_{i i}=O\left(N^{-1}\right), b_{i \neq j}=O\left(N^{-2}\right)$, and $a_{i}+b_{i i} M_{i}=O\left(N^{-1}\right)$, for $i, j=1, \ldots, m$. This claim is proved by induction. First, notice that $D$ has the same $m \times m$ block structure as in Eq. (A4) except all its parameters are of order 1. Using the fact

$$
\left(a+b \mathbb{I}_{M M}\right)^{-1}=\frac{1}{a}-\frac{b}{a(b M+a)} \mathbb{I}_{M M}
$$

and Eq. (A3), we find that, for $m=2, D^{-1}$ indeed has the properties described by Eq. (A4). Then assume the claim is true for $m=n-1$. By straightforward calculation using Eqs. (A3) and (A5), we find the claim is also true for $m=n$.

Step 3. Substituting Eqs. (A4) to (A2) and using Eq. (2.11), we get Eq. (A1). This completes the proof.
[1] K. Rajan and L. F. Abbott, Phys. Rev. Lett. 97, 188104 (2006).

[2] H. Sompolinsky, A. Crisanti, and H. J. Sommers, Phys. Rev. Lett. 61, 259 (1988).
[3] M. A. Stephanov, Phys. Rev. Lett. 76, 4472 (1996).

[4] J. J. M. Verbaarschot and T. Wettig, Annu. Rev. Nucl. Part. Sci. 50, 343 (2000). 
[5] J. J. M. Verbaarschot, H. A. Weidenmüller, and M. R. Zirnbauer Phys. Rep. 129, 367 (1985).

[6] H. J. Sommers, A. Crisanti, H. Sompolinsky, and Y. Stein, Phys. Rev. Lett. 60, 1895 (1988).

[7] V. L. Girko, Theory Probab. Its Appl. 29, 694 (1984).

[8] G. M. Shepherd et al., The Synaptic Organization of the Brain (Oxford University Press, London, 2004).

[9] C. Holmgren, T. Harkany, B. Svennenfors, and Y. Zilberter, J. Physiol. 551, 139 (2003).

[10] J. Ginibre, J. Math. Phys. 6, 440 (1965).

[11] M. N. Shadlen and W. T. Newsome, Curr. Opin. Neurobiol. 4, 569 (1994).

[12] T. W. Troyer and K. D. Miller, Neural Comput. 9, 971 (1997).

[13] B. Barbour, N. Brunel, V. Hakim, and J. P. Nadal, Trends Neurosci. 30, 622 (2007).

[14] S. Song, P. J. Sjöström, M. Reigl, S. B. Nelson, and D. B. Chklovskii, PLoS Biol. 3, 507 (2005).
[15] E. Brézin, S. Hikami, and A. Zee, Phys. Rev. E 51, 5442 (1995).

[16] A. Zee, Nucl. Phys. B 474, 726 (1996).

[17] T. Tao, arXiv:1012.4818 [Probab. Theory Relat. Fields (to be published)].

[18] A. Pizzo, D. Renfrew, and A. Soshnikov, arXiv:1103.3731 [Ann. Inst. Henri Poincaré, Sect. B (to be published)].

[19] K. Zyczkowski and H.-J. Sommers, J. Phys. A 33, 2045 (2000).

[20] Y. Wei and Y. V. Fyodorov, J. Phys. A 41, 502001 (2008).

[21] E. Bogomolny, J. Phys. A 43, 335102 (2010).

[22] R. A. Janik, M. A. Nowak, G. Papp, and I. Zahed, Nucl. Phys. B 501, 603 (1997).

[23] J. Feinberg and A. Zee, Nucl. Phys. B 504, 579 (1997).

[24] J. Feinberg and A. Zee, Nucl. Phys. B 501, 643 (1997).

[25] J. Feinberg, R. Scalettar, and A. Zee, J. Math. Phys. 42, 5718 (2001).

[26] J. Feinberg, J. Phys. A 39, 10029 (2006). 